\title{
Growth Dynamics of Photoinduced Phase Domain in Cyano-Complex Studied by Boundary Sensitive Raman Spectroscopy
}

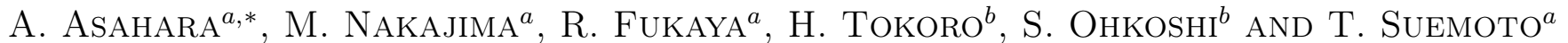 \\ ${ }^{a}$ Institute for Solid State Physics, The University of Tokyo, Kashiwanoha 5-1-5, Kashiwashi, Chiba, 277-8581, Japan \\ ${ }^{b}$ Department of Chemistry, School of Science, The University of Tokyo \\ 7-3-1 Hongo, Bunkyo-ku Tokyo, 113-0033, Japan
}

\begin{abstract}
Rubidium manganese hexacyanoferrate exhibits charge transfer phase transition from high temperature phase to low temperature phase at $230 \mathrm{~K}$. This phase transition can also be triggered by light irradiation resonantly inducing charge transfer from $\mathrm{Mn}^{2+}$ to $\mathrm{Fe}^{3+}$. In the present study, boundary sensitive Raman spectroscopy was performed for $\mathrm{Rb}_{0.94} \mathrm{Mn}\left[\mathrm{Fe}(\mathrm{CN})_{6}\right]_{0.98} \cdot 0.2 \mathrm{H}_{2} \mathrm{O}$ in both cases of photoinduced and thermal phase transition. Since the frequencies of $\mathrm{C} \equiv \mathrm{N}$ stretching vibration modes are very sensitive to the valence states of the adjacent metal ions, we can quantify the distribution of not only high and low temperature phase but also boundary configurations from the observed spectra. We obtained the time evolution of the fraction ratios of the valence states from the observed peak areas. In the case of photoinduced situation, the boundary increases up to $15 \%$ when high temperature phase diminishes to $55 \%$ of the initial fraction. This is quite different from the result in thermal phase transition where the boundary is created only $0.8 \%$ at the same high temperature phase fraction value. We conclude that many small domains are preferably created in photoinduced phase transition since the ratio of boundary is large, while large domains grow in thermal phase transition.
\end{abstract}

PACS: 64.60.-i, 82.50.Hp, 78.30.-j

\section{Introduction}

Recently, photoinduced phase transition is one of attractive research fields in material science. However, there are many unsolved subjects particularly about the domain dynamics. For example, the position where the phase transition starts and the photocreated domain size and so on have not been clearly understood. There are some theoretical approaches to understand them $[1,2]$. Investigating the domain dynamics experimentally is very significant to understand the mechanism of phase transitions in detail. In this research, we aimed to observe such domain growth dynamics in a cyano-complex.

Cyano-complexes exhibit various interesting optical responses. Particularly, rubidium manganese hexacyanoferrate is an attractive material because of its fascinating multiple functionalities. For instance, a pressure-induced magnetic pole inversion [3], coexistence of ferroelectricity and ferromagnetism [4], electric field induced charge transfer phase transition [5], and reversible photoinduced phase transition at low temperature [6] have been reported. This material shows thermal phase transition from high temperature phase (HTP) to low temperature phase (LTP) at the phase transition tempera-

* corresponding author; e-mail: asahara@issp.u-tokyo.ac.jp ture $\left(T_{1 / 2 \downarrow}=230 \mathrm{~K}\right)$ in cooling process. In HTP, $\mathrm{Fe}^{3+}$ and $\mathrm{Mn}^{2+}$ metal ions are bridged by a $\mathrm{CN}^{-}$ion to construct $\mathrm{Fe}^{3+}-\mathrm{CN}-\mathrm{Mn}^{2+}$ valence configuration. When the thermal phase transition occurs, charges on Mn sites are transferred to $\mathrm{Fe}$ sites to create $\mathrm{Fe}^{2+}-\mathrm{CN}-\mathrm{Mn}^{3+}$ configuration. This is the basic configuration of LTP. This charge transferred phase transition can also be triggered by light irradiation at room temperature. Irradiation with $400 \mathrm{~nm}$ light stimulates ligand-to-metal charge transfer (LMCT) band to induce charge transfer from $\mathrm{CN}^{-}$to $\mathrm{Fe}^{3+}[7]$. Then, an electron is supplied from $\mathrm{Mn}^{2+}$ to $\mathrm{CN}$ group and $\mathrm{Fe}^{2+}-\mathrm{CN}-\mathrm{Mn}^{3+}$ configuration in LTP appears. In this way, the charge transferred photoinduced phase transition is accomplished.

The degree of progress of this phase transition can be quantitatively verified by observing $\mathrm{C} \equiv \mathrm{N}$ vibration modes by the Raman scattering and IR absorption spectroscopy $[8,9]$. Since the frequency of $\mathrm{C} \equiv \mathrm{N}$ stretching vibration mode is very sensitive to the valence states of the adjacent metal ions, we can get information about valence distribution of the sample from the ratio of the spectral intensity. In addition, it is reported that we can observe $\mathrm{Fe}^{3+}-\mathrm{CN}-\mathrm{Mn}^{3+}$ peak by resonance Raman effect by using light whose wavelength corresponds to LMCT band [8]. This valence configuration of $\mathrm{Fe}^{3+}{ }_{-} \mathrm{CN}-$ $\mathrm{Mn}^{3+}$ corresponds to the phase boundary which appears at the interface between HTP and LTP. We can utilize 
this property to quantify the distribution of not only the inside of domain but also the boundary. It is expected that we can get revolutionary information about the domain growth dynamics in phase transition.

In the present study, we investigated the domain growth dynamics in phase transition for rubidium manganese hexacyanoferrate by applying the boundary sensitive Raman spectroscopy. In particular, we focused on differences of the domain dynamics between photoinduced and thermal phase transition.

\section{Experimental}

In the present study, the Raman scattering spectroscopy with $442 \mathrm{~nm}$ continuous wave (CW) light was performed for a powdered sample whose compositional formula is $\mathrm{Rb}_{0.94} \mathrm{Mn}\left[\mathrm{Fe}(\mathrm{CN})_{6}\right]_{0.98} \cdot 0.2 \mathrm{H}_{2} \mathrm{O}$ as shown in Fig. 1. A helium-cadmium-ion $\mathrm{CW}$ laser (KIMMON) whose wavelength is $442 \mathrm{~nm}$ was applied as a light source, which works as an incident light of the Raman spectroscopy and a pump light to trigger the photoinduced phase transition simultaneously. Since the selected wavelength is appropriately far from the center of LMCT band at $405 \mathrm{~nm}$, we could realize a good experimental condition that the speed of photoinduced phase transition is moderately slow and the peak corresponding to $\mathrm{Fe}^{3+}$ $\mathrm{CN}-\mathrm{Mn}^{3+}$ is detectable by the resonant Raman effect. The sample dispersed in liquid paraffin was put between two sheets of sapphire plates and placed in a He-gas-flow type cryostat. The incident light is focused on the sample, whose spot diameter is $200 \mu \mathrm{m}$, and the scattered light from the sample was collected into a triple grating polychromator (SPEX 1877). The dispersed light was detected by a liquid-nitrogen-cooled charge coupled device (Horiba Jobin Yvon, Symphony) equipped with the polychromator.

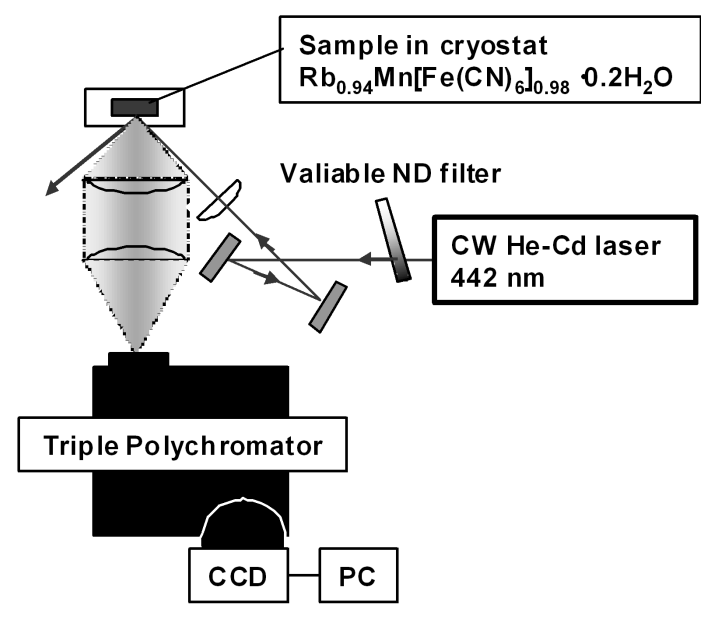

Fig. 1. Experimental setup.

Firstly, we investigated the transition dynamics in the case of photoinduced phase transition at room temperature. The spectra were measured at every one minute while the pump light was irradiated to the sample all the time. The excitation density is $4.8 \mathrm{~W} / \mathrm{cm}^{2}$. Secondly, the Raman spectra were measured at various temperatures around $T_{1 / 2 \downarrow}$ at $230 \mathrm{~K}$ in cooling process. These all spectra were collected within initial two minutes from the light irradiation whose excitation density is $12.1 \mathrm{~W} / \mathrm{cm}^{2}$, when the photoinduced effect is negligible. The beam spot was moved to a fresh location in the sample at every scan.

\section{Results and discussion}

Figure $2 \mathrm{a}$ shows the time evolutions of the Raman spectra in photoinduced phase transition. As the transition evolves, the peak at $2170 \mathrm{~cm}^{-1}$ corresponding to HTP gradually decreases. Instead, the peaks at 2096 and $2137 \mathrm{~cm}^{-1}$ corresponding to LTP and at $2234 \mathrm{~cm}^{-1}$ corresponding to boundary appear. A laser heating effect can be neglected here, since a heating does not create LTP from HTP. Figure $2 \mathrm{~b}$ shows the temperature dependence of the Raman spectra in thermal phase transition. When the temperature is lowered, the peak at $2170 \mathrm{~cm}^{-1}$ due to HTP gradually decreases and the two peaks at 2099 and $2117 \mathrm{~cm}^{-1}$ due to LTP appear from $240 \mathrm{~K}$. The peak due to $\mathrm{Fe}^{3+}-\mathrm{CN}-\mathrm{Mn}^{3+}$ at $2206 \mathrm{~cm}^{-1}$ appears from around $230 \mathrm{~K}$, though the intensity is much smaller than that in the case of photoinduced phase transition as shown in Fig. 2a.

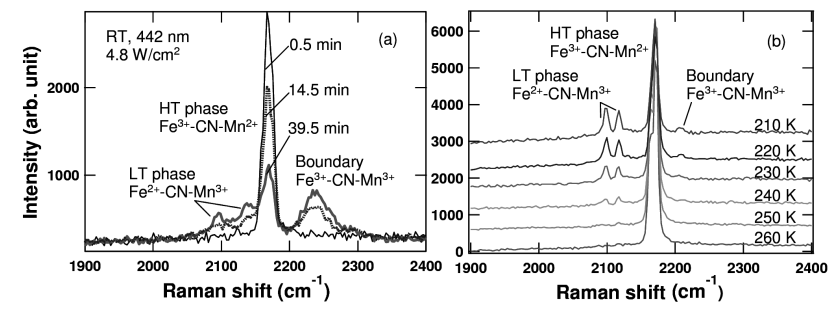

Fig. 2. Raman spectra of (a) the time evolutions in photoinduced phase transition and of (b) the temperature dependence in thermal phase transition.

The assignments of these peaks were fundamentally based on empirically obtained relations between $\mathrm{C} \equiv \mathrm{N}$ vibration's frequency and valence states of the adjacent metal ions. The frequencies for $\mathrm{Fe}^{2+}-\mathrm{CN}-\mathrm{M}^{2+}, \mathrm{Fe}^{2+}$ $\mathrm{CN}-\mathrm{M}^{3+}, \mathrm{Fe}^{3+}-\mathrm{CN}-\mathrm{M}^{2+}$ and $\mathrm{Fe}^{3+}-\mathrm{CN}-\mathrm{M}^{3+}(\mathrm{M}=$ transition metal) have been reported to typically correspond to the ranges of 2065-2110, 2090-2140, 2146-2185 and $2180-2210 \mathrm{~cm}^{-1}$ in the Raman and infrared spectrum, respectively $[10,11]$. Therefore, the peaks at 2170 and $2234 \mathrm{~cm}^{-1}$ in Fig. 2a are assigned to HTP and $\mathrm{Fe}^{3+}-\mathrm{CN}-$ $\mathrm{Mn}^{3+}$, respectively. The peaks at 2170 and $2206 \mathrm{~cm}^{-1}$ in Fig. $2 \mathrm{~b}$ are also assigned to $\mathrm{HTP}$ and $\mathrm{Fe}^{3+}-\mathrm{CN}-\mathrm{Mn}^{3+}$, respectively.

Since the frequency ranges corresponding to $\mathrm{Fe}^{2+}-\mathrm{CN}-$ $\mathrm{Mn}^{2+}$ and LTP have some overlapping, we must be careful to distinguish these two species. The peaks at 2099 and $2117 \mathrm{~cm}^{-1}$ in Fig. $2 \mathrm{~b}$ can be assigned to LTP, since 
a previous report by Cobo et al. [12] clearly assigned two sharp peaks at 2096 and $2114 \mathrm{~cm}^{-1}$ to LTP.

Furthermore, Vertelman et al. [10] and Lummen et al. [11] have observed several broadened peaks at 2086$2130 \mathrm{~cm}^{-1}$ in the Raman spectra and assigned them to LTP. Based on these studies, we attributed the peaks at 2096 and $2137 \mathrm{~cm}^{-1}$ in Fig. 2a to LTP, though the spectral shapes and frequencies are somewhat different. The differences are probably due to several factors specific to the photoinduced phase transition.

Upward frequency shifts more than $10 \mathrm{~cm}^{-1}$ have been commonly observed especially at the early stage of the photoinduced transition from HTP to LTP, whose mechanism was explained by Fukaya et al. [8] in detail. This large shift can be ascribed to the considerable strain fields caused by lattice mismatch between the optically created metastable LTP domains and the surrounding HTP framework. The broadening of the peaks was ascribed to the peak splitting due to decreased symmetry of the crystal (local tetragonal deformation of lattice). Such a broadening caused by reduced crystal symmetry has been observed, when disorder is increased in the samples deviated from the stoichiometry [12]. Although some contribution from $\mathrm{Fe}^{2+}-\mathrm{CN}-\mathrm{Mn}^{2+}$ might be included, the main part of these two peaks can be assigned to LTP.

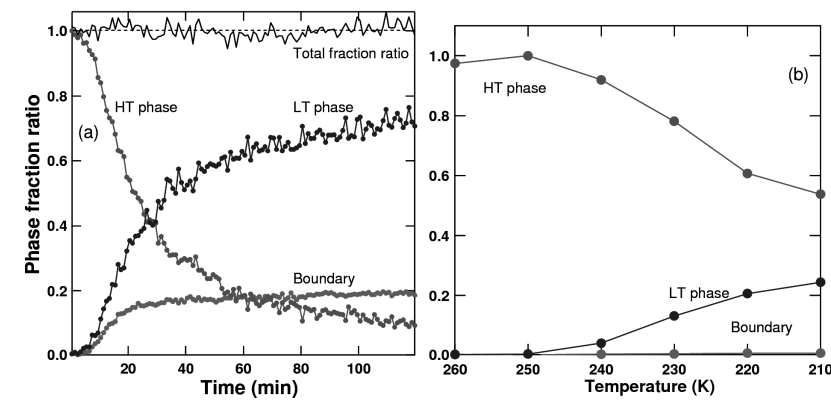

Fig. 3. The evolutions of HTP (red), LTP (blue) and boundary (green) in (a) photoinduced phase transition and (b) thermal phase transition.

As shown in Fig. 3a, we estimated the time evolution of the each fraction ratios from the observed peak areas in Fig. 2a in the case of photoinduced phase transition. The peak areas were evaluated from a multi-Lorentzian fitting analysis of the observed spectra. Though the scattering cross-sections of these peaks are unclear, we determined the relative ratios by assuming that sum of the fraction ratios remains constant. Here, the fraction of $\mathrm{Fe}^{2+}-\mathrm{CN}-$ $\mathrm{Mn}^{2+}$ which was not observed is assumed to be equal to that of $\mathrm{Fe}^{3+}-\mathrm{CN}-\mathrm{Mn}^{3+}$. Since the total fraction ratio (black) keeps nearly constant as shown in Fig. 3a, the determined ratios are valid at all stages in this transition. In Fig. 3a, HTP (red) decreases and LTP (blue) increases symmetrically and boundary (green) increases up to $20 \%$ at 120 min.

The evolution in thermal phase transition is also estimated in Fig. 3b. Now, we applied the same relative ratios of scattering cross-sections used in the case of photoinduced situation. In this figure, HTP (red) and LTP (blue) behave like in the case of photoinduced phase transition, though the thermal transition is not accomplished completely. However, as for boundary (green), there is a definite difference. For example, when HTP diminishes to about $55 \%$ of the initial fraction at $210 \mathrm{~K}$, the boundary is created only $0.8 \%$. This is quite different from the result in the case of photoinduced phase transition where the boundary is created about $15 \%$ at the same HTP fraction value at $20 \mathrm{~min}$, while LTP fraction is $25 \%$ and it is almost the same value in both cases.

Generally speaking, as the averaged domain size becomes smaller, the amount of boundary configuration increases. Consequently, we conclude that many small domains are preferably created in photoinduced phase transition since the ratio of boundary is large, while a few large domains grow in thermal phase transition. This result is interpreted as follows. In the case of photoinduced phase transition, nucleation of domain occurs anywhere in the sample because the excitation energy is sufficiently high. While in the case of thermal phase transition, such occurrence of nucleation is restricted on places where charge transfers are easy to occur because of the lower energy required for the transition, for example on defects.

\section{Conclusion}

The domain growth dynamics of a phase transition (HTP to LTP) in rubidium manganese hexacyanoferrate was investigated by boundary sensitive Raman spectroscopy in both cases of photoinduced phase transition and thermal one. We estimated the time evolution of each fractions (HTP, LTP and boundary) from the observed spectra, and successfully found that when the amount of HTP diminishes to $55 \%$ of that in initial state, boundary is created about $15 \%$ in the case of photoinduced phase transition while only $0.8 \%$ in the case of thermal transition. We interpreted this difference as following from the viewpoint of the size of created domains. In the case of photoinduced phase transition many small sized domains are created, while in the case of thermal phase transition a few large sized domains grow.

\section{Acknowledgments}

This work was supported by the Grant-in Aid for Scientific Researches (B) from the Ministry of Education, Culture, Sports, Science, and Technology of Japan and the author was supported by Global COE Program "the Physical Sciences Frontier", MEXT, Japan. The authors thank M. Nishino (NIMS) for discussions.

\section{References}

[1] R. Yabuki, K. Nasu, Phys. Lett. A 320, 289 (2004).

[2] M. Nishino, C. Enachescu, S. Miyashita, K. Boukheddaden, F. Varret, Phys. Rev. B 82, 020409 (2010). 
[3] L. Egan, K. Kamenev, D. Papanikolaou, Y. Takabayashi, S. Margadonna, J. Am. Chem. Soc. 128, 6034 (2006).

[4] S. Ohkoshi, H. Tokoro, T. Matsuda, H. Takahashi, H. Irie, K. Hashimoto, Angew. Chem. Int. Ed. 46, 3238 (2007)

[5] T. Mahfoud, G. Molnar, S. Bonhommeau, S. Cobo, L. Salmon, P. Demont, H. Tokoro, S. Ohkoshi, K. Boukheddaden, A. Bousseksou, J. Am. Chem. Soc. 131, 15049 (2009).

[6] H. Tokoro, T. Matsuda, T. Nuida, Y. Moritomo, K. Ohoyama, E.D.L. Dangui, K. Boukheddaden, S. Ohkoshi, Chem. Mater. 20, 423 (2008).

[7] E.D. Loutete-Dangui, E. Codjovi, H. Tokoro, P.R. Dahoo, S. Ohkoshi, K. Boukheddaden, Phys. Rev. B 78, 014303 (2008).

[8] R. Fukaya, M. Nakajima, H. Tokoro, S. Ohkoshi, T. Suemoto, J. Chem. Phys. 131, 154505 (2009).
[9] S. Ohkoshi, H. Tokoro, K. Hashimoto, Coord. Chem. Rev. 249, 1830 (2005).

[10] E.J.M. Vertelman, T.T.A. Lummen, A. Meetsma, M.W. Bouwkamp, G. Molnar, P.H.M. van Loosdrecht, P.J. van Koningsbruggen, Chem. Mater 20, 1236 (2008).

[11] T.T.A. Lummen, R.Y.N. Gengler, P. Rudolf, F. Lusitani, E.J.M. Vertelman, P.J. van Koningsbruggen, M. Knupfer, O. Molodtsova, J.-J. Pireaux, P.H.M. van Loosdrecht, J. Phys. Chem. C 112, 14158 (2008).

[12] S. Cobo, R. Fernandez, L. Salmon, G. Molnar, A. Bousseksou, Eur. J. Inorg. Chem. 11, 1549 (2007). 